\title{
A New Method to Assess Entrepreneurship Competence in University Students Using Based on Plithogenic Numbers and SWOT Analysis
}

\author{
Noel Batista Hernández ${ }^{1}$, Maikel Y. Leyva Vázquez² ${ }^{2}$, Erick González \\ Caballero $^{3}$, Lilia E. Valencia Cruzaty ${ }^{4}$, Wilmer Ortega Chávez ${ }^{5}$, and Florentin \\ Smarandache ${ }^{6}$ \\ ${ }^{1}$ Universidad de Guayaquil, Guayaquil, Ecuador \\ ${ }^{2}$ Universidad Politécnica Salesiana, Guayaquil, Ecuador \\ ${ }^{3}$ Center of Mathematics, Technological University of Havana, Havana, Habana, Cuba \\ ${ }^{4}$ Universidad Estatal Península de Santa Elena, Santa Elena, Ecuador \\ ${ }^{5}$ Universidad Nacional Intercultural de la Amazonia, Ucayali, Peru \\ ${ }^{6}$ Math \& Science Department, University of New Mexico, Gallup, NM, USA
}

\begin{abstract}
This study introduces a method to measure the implementation of the training strategy of entrepreneurship competence as a contribution to the integrity and quality of future graduates from any higher education institutions. For this purpose, the theory of plithogenic sets was applied. These sets were defined to model concepts arising from the dynamic interaction of other simpler ones, which may have contradictions among each other and include neutralities or indeterminacies. To the best of our knowledge, this is the first time that plithogenic sets are applied in the mathematical modeling of entrepreneurship competence or any other pedagogic competence. The plithogenic numbers are represented and applied to SWOT (Strengths, Weaknesses, Opportunities, and Threats) analysis. This method was successfully applied at the University of Guayaquil, Ecuador. It is a simple method that includes an assessment based on linguistic terms and indeterminacies.
\end{abstract}

Keywords: Entrepreneurship competence, Plithogenic logic, SWOT analysis

\author{
Received: Mar. 27, 2021 \\ Revised : Jun. 5, 2021 \\ Accepted: Jun. 9, 2021 \\ Correspondence to: Noel Batista-Hernández \\ (noelbatista1965@gmail.com) \\ (CThe Korean Institute of Intelligent Systems
}

(c) This is an Open Access article distributed under the terms of the Creative Commons Attribution Non-Commercial License http://creativecommons.org/licenses/by-nc/ 3.0/) which permits unrestricted noncommercial use, distribution, and reproduction in any medium, provided the original work is properly cited.

\section{Introduction}

Evaluating the feasibility of executing the strategy for the development of entrepreneurship competence among students belongs to the teleology of university education, which gathers relevance and opportunity as facilitating categories to increase critical lateral thinking. It establishes an active attitude in the students and increases their capacity to generate prosperity and strengths that influence an individual's performance collection [1-4].

In this article, we understand entrepreneurship as a complex system of knowledge, skills, attitudes, and values that interact synergistically and enable the self-appointed and effective performance of the individual, by providing them with tools to create, manage, interpret, understand, and transform the social environment with a critical, proactive, and innovative vision, sustaining a model of life, of present and future personal development from the 
educational context [2[5|6].

Pedagogical entrepreneurship competence is assessed in this systematization, which is not restricted to the narrow margins of an individual building; it instead interacts with social demands, the claim to their various attributes, and the need to materialize an attitude that is conducive to general welfare, in the present as well as in the future [78].

To analyze feasibility in general, we used the method of SWOT (Strengths, Weaknesses, Opportunities, and Threats) analysis that aggregates the relationships between the different parameters and the weights of the quadrants, which allowed us to achieve higher accuracy in the calculation and determination of the strategic moment, and the feasibility of applying transformation actions in the training of higher-education students [910]. By approaching the concept of entrepreneurship competence through the idea of the system allows for the application of mathematical-logical modeling based on plithogenic sets; in addition to that, this type of combination allows the inclusion of indeterminacy [11-13].

Plithogeny is the genesis or origin, creation, formation, development, and evolution of new entities from dynamic and organic mergers of multiple contradictory and/or neutral and/or non-contradictory old entities. Plithogeny advocates the connections and unification of theories and ideas in any field. We can consider "knowledge" as "entities" in various fields, such as the soft sciences, hard sciences, and theories of the arts and letters [11].

Therefore, plithogeny is the dynamics of many types of opposites and/or their neutrals and/or non-opposites and their organic fusion. Plithogeny is a generalization of dialectics (dynamics of a kind of opposite: $\langle A\rangle$ and $<$ anti $A\rangle$ ), the neutrosophy (dynamics of a kind of opposites and their neutrals: $\langle A\rangle$ and $\langle$ anti $A\rangle$, and $\langle$ neut $A\rangle$ ), because plithogeny studies the dynamics of many types of opposites and their neutral and non-opposites ( $<A>$ and $<$ anti $A>$, and $<$ neut $A>$, $<B>$ and $\langle$ anti $B>$ and $<$ neut $B>$, etc.), and many that are not opposites ( $\langle C\rangle,\langle D\rangle$, etc.) altogether. A particular application and concept derived from plithogeny is the plithogenic set, which is an extension of the classical set, fuzzy set, intuitionist fuzzy set, and neutrosophic set, and has many scientific applications [11].

A plithogenic set $\mathrm{P}$ is a set whose elements are characterized by one or more attributes, and each attribute can have many values. Until now, these have mainly been applied in decisionmaking mathematical models [13|14]. In this research, we will use them to determine the foundation and structure of a solution to the formation of entrepreneurship competence in students. This new theory fits the purpose of this article as it is a tool to represent complex, systemic, and dynamic concepts with degrees of relatedness, where different simpler concepts interact with each other. To the best of our knowledge, this is the first time that plithogenic sets are used to measure the phenomenon of psycho-pedagogy competence [11].

In this case, plithogenic numbers are obtained from the assessment of a group of experts, who evaluate the situation of entrepreneurship competence in the students of any higher education institution, where the University of Guayaquil is used as a case study. The assessments were based on the SWOT analysis, and the plithogenic numbers were input into a SWOT matrix. However, experts do not directly use plithogenic numbers in their evaluations; they evaluate by using linguistic terms, which are associated with plithogenic numbers and are used for the SWOT evaluation of the results. Some background of hybridization of SWOT analysis with neutrosophy and other methods can be found in [6 14 [15].

This paper is divided into the following sections: Section 2 presents the main concepts of plithogenic sets and SWOT analysis. Section 3 contains the material and methods where we introduce the method proposed in this paper, and Section 4 covers the study we conducted on the entrepreneurship competence of students at the University of Guayaquil using the proposed method. Section 5 presents the discussion, and the last section concludes the paper.

\section{Preliminary Concepts}

This section is divided into two subsections: the first one summarizes the main concepts of plithogenic sets, whereas the second one discusses the concepts of SWOT analysis.

\subsection{Basic Notions of Plithogenic Sets}

Let $U$ be a universe of discourse, and $P$ be a non-empty set of elements, $P \subseteq U$. Let A be a non-empty set of uni-dimensional attributes $A=\left\{\alpha_{1}, \alpha_{2}, \cdots, \alpha_{m}\right\}, m \geq 1$, and $\alpha_{i} \in A$ is a given attribute whose spectrum of all the possible values (or states) is the non-empty set $S$, where $S$ can be a set of finite discrete, $S=\left\{s_{1}, s_{2}, \cdots, s_{l}\right\}, 1 \leq l<\infty$, or infinitely numerable set $S=\left\{s_{1}, s_{2}, \cdots, s_{\infty}\right\}$, or an infinitely uncountable set (continuous), $S=] a, b[, a<b$, where $] \cdots[$ is any open, semi-open, or a closed interval set of real numbers or another set. 
Let $V$ be a non-empty subset of $S$, where $V$ is the range of all attribute values required by experts for the application. Each element $x \in P$ is characterized by the values of all attributes in $V=\left\{v_{1}, v_{2}, \cdots, v_{n}\right\}$, for $n \geq 1$.

In the set of values of the attribute, $V$, in general, there is a dominant attribute value, which is determined by experts in its application. The dominant attribute value is the most important attribute value that experts are interested in.

Each attribute value $v_{i} \in V$ has a corresponding degree of appurtenance $d(x, v)$ of the element $x$ to the set $P$ with respect to certain criteria.

The degree of appurtenance can be either a fuzzy degree of appurtenance, an intuitionistic fuzzy degree of appurtenance, or a neutrosophic degree of appurtenance to the plithogenic set.

Thus, the attribute value appurtenance degree function is:

$$
d: P \times V \rightarrow \mathcal{P}\left([0,1]^{z}\right), \forall(x, v) \in(P, V)
$$

Thus $d(x, v)$ is a subset of $[0,1]^{z}$, where $\mathcal{P}\left([0,1]^{z}\right)$ is the power set of $[0,1]^{z}$, where $z=1$ (fuzzy degree of appurtenance), $z=2$ (intuitionistic fuzzy degree of appurtenance), and $z=3$ (neutrosophic degree of appurtenance).

Let $|V| \geq 1$ be a cardinality. Let $c: V \times V \rightarrow[0,1]$ be the attribute value contradiction degree function between any two attribute values $v_{1}$ and $v_{2}$, denoted by $c\left(v_{1}, v_{2}\right)$, and satisfy the following axioms:

1. $c\left(v_{1}, v_{1}\right)=0$, the degree of contradiction between the same attribute value is zero,

2. $c\left(v_{1}, v_{2}\right)=c\left(v_{2}, v_{1}\right)$, commutativity.

We can define a fuzzy attribute value contradiction degree function ( $c$ as before, we denote by $c_{F}$ to distinguish it from the following two), an intuitionistic fuzzy attribute value contradiction degree function $\left(c_{I F}: V \times V \rightarrow[0,1]^{2}\right)$, or more generally, a neutrosophic attribute value contradiction degree function $\left(c_{N}: V \times V \rightarrow[0,1]^{3}\right)$, which can be used to increase the complexity of the calculation, but also to increase the accuracy.

We calculate the degree of contradiction between the values of the unidimensional attributes. Multi-dimensional attribute values can be divided into their corresponding one-dimensional attribute values.

The attribute value contradiction degree function helps plithogenic aggregation operators and plithogenic inclusion (partial order) to obtain a more accurate result.

The attribute value contradiction degree function is designed in each field, where a plithogenic set is used according to the application to be solved. If it is ignored, the aggregations still work, but the result may lose accuracy.

Thus, $(P, a, V, d, c)$ is called a plithogenic set, [11 16]:

1. Where $P$ is a set, $a$ is an attribute (multi-dimensional in general), $V$ is the range of values of the attribute, $d$ is the degree of appurtenance of the attribute value of each element $x$ to the set $P$ for some given criteria $(x \in P)$, and $d$ means $d_{F}$ or $d_{I F}$ or $d_{N}$, when it is a degree of fuzzy appurtenance, an intuitionistic fuzzy appurtenance, or a degree of neutrosophic appurtenance, respectively, of an element $x$ to the plithogenic set $P$;

2. $c$ means $c_{F}$ or $c_{I F}$ or $c_{N}$, when it is a fuzzy attribute value contradiction degree function, intuitionistic fuzzy attribute value contradiction degree function, or neutrosophic attribute value contradiction degree function, respectively.

Functions $d(\cdot, \cdot)$ and $c(\cdot, \cdot)$ are defined according to the applications that experts need to solve.

Then, the following notation is used: $x(d(x, V))$, where $d(x, V)=\{d(x, v)$, for every $v \in V\}, \forall x \in P$.

The attribute value contradiction degree function is calculated between each attribute value concerning the dominant attribute value (denoted by $v_{D}$ ) in particular, and for other attribute values as well.

The attribute value contradiction degree function $c$ evaluated between the values of two attributes is used in the definition of plithogenic aggregation operators - intersection (AND), union (OR), implication $(\Rightarrow)$, equivalence $(\Leftrightarrow)$, inclusion (partial order), and other plithogenic aggregation operators that combine two or more degrees of attribute values based on a $t$-norm and a $t$-conorm [17]).

Most plithogenic aggregation operators are linear combinations of one fuzzy t-norm (denoted by $\wedge_{F}$ ) with one fuzzy t-conorm (denoted by $\vee_{F}$ ); however, nonlinear combinations can also be constructed.

If the $t$-norm is applied over the dominant attribute value denoted by $v_{D}$, and the contradiction between $v_{D}$ and $v_{2}$ is $c\left(v_{D}, v_{2}\right)$, then attribute value $v_{2}$ is applied as follows:

$$
\left[1-c\left(v_{D}, v_{2}\right)\right] \cdot t_{n o r m}\left(v_{D}, v_{2}\right)+c\left(v_{D}, v_{2}\right) \cdot t_{\text {conorm }}\left(v_{D}, v_{2}\right)
$$

Or, by using symbols:

$$
\left[1-c\left(v_{D}, v_{2}\right)\right] \cdot\left(v_{D} \wedge_{F} v_{2}\right)+c\left(v_{D}, v_{2}\right) \cdot\left(v_{D} \vee_{F} v_{2}\right)
$$


Similarly, if the $t$-conorm is applied to the dominant attribute value denoted by $v_{D}$, and the contradiction between $v_{D}$ and $v_{2}$ is $c\left(v_{D}, v_{2}\right)$, then on the attribute value $v_{2}$ is applied:

$\left[1-c\left(v_{D}, v_{2}\right)\right] \cdot t_{c o n o r m}\left(v_{D}, v_{2}\right)+c\left(v_{D}, v_{2}\right) \cdot t_{n o r m}\left(v_{D}, v_{2}\right)$

Or, by using symbols:

$$
\left[1-c\left(v_{D}, v_{2}\right)\right] \cdot\left(v_{D} \vee_{F} v_{2}\right)+c\left(v_{D}, v_{2}\right) \cdot\left(v_{D} \wedge_{F} v_{2}\right)
$$

The plithogenic neutrosophic intersection is defined as:

$$
\begin{aligned}
& \left(a_{1}, a_{2}, a_{3}\right) \wedge_{P}\left(b_{1}, b_{2}, b_{3}\right) \\
& =\left(a_{1} \wedge_{F} b_{1}, \frac{1}{2}\left[\left(a_{2} \wedge_{F} b_{2}\right)+\left(a_{2} \vee_{F} b_{2}\right)\right], a_{3} \vee_{F} b_{3}\right)
\end{aligned}
$$

The plithogenic neutrosophic union is defined as:

$$
\begin{aligned}
& \left(a_{1}, a_{2}, a_{3}\right) \vee_{P}\left(b_{1}, b_{2}, b_{3}\right) \\
& =\left(a_{1} \vee_{F} b_{1}, \frac{1}{2}\left[\left(a_{2} \wedge_{F} b_{2}\right)+\left(a_{2} \vee_{F} b_{2}\right)\right], a_{3} \wedge_{F} b_{3}\right) .
\end{aligned}
$$

In other words, concerning what applies to membership, the opposite applies to non-membership, whereasile in indeterminacy, the average between them applies.

The plithogenic neutrosophic inclusion is defined as follows:

As the degrees of contradiction are $c\left(a_{1}, a_{2}\right)=c\left(a_{2}, a_{3}\right)=$ $c\left(b_{1}, b_{2}\right)=c\left(b_{2}, b_{3}\right)=0.5$, it applies $a_{2} \geq\left[1-c\left(a_{1}, a_{2}\right)\right] b_{2}$ or $a_{2} \geq(1-0.5) b_{2}$ or $a_{2} \geq 0.5 b_{2}$, while $c\left(a_{1}, a_{3}\right)=c\left(b_{1}, b_{3}\right)=$ 1 .

Having $a_{1} \leq b_{1}$, the opposite is fulfilled for $a_{3} \geq b_{3}$; hence $\left(a_{1}, a_{2}, a_{3}\right) \leq_{P}\left(b_{1}, b_{2}, b_{3}\right)$ if and only if $a_{1} \leq b_{1}, a_{2} \geq 0.5 b_{2}$, and $a_{3} \geq b_{3}$.

\subsection{SWOT Analysis}

The SWOT analysis is a methodology for studying the situation of a company or a project by analyzing its internal characteristics (weaknesses and strengths) and its external situation (threats and opportunities) in a matrix [9]10].

The analysis consists of four steps:

1. External analysis,

2. Internal analysis,

3. Preparation of the SWOT matrix,
4. Determination of the strategy to be used.

The organization does not exist and cannot exist outside the environment that surrounds it, and thus, external analysis allows us to identify the opportunities and threats that the context may present to an organization. These are the components of external analysis. Opportunities are those positive factors that are generated in the environment and that, once identified, the institution can take advantage of. Threats are negative situations, external to the program or project, that can attack it, and hence, when appropriate, it may be necessary to design the right strategy to circumvent them.

The internal elements to be analyzed during the SWOT analysis correspond to the strengths and weaknesses of the availability of capital resources, personnel, assets, product quality, internal and market structure, and consumer perception, among others. The internal analysis allows fixing the strengths and weaknesses of the organization, carrying out a study that allows understanding the quantity and quality of the resources and processes with which the organization has.

Strengths are all internal and positive elements that differentiate the program or project from others of the same kind. Weaknesses relate to all those elements, energy resources, skills, and assets that the organization already has and that constitute barriers to the good performance of the organization. Weaknesses are internal problems that once identified should be eliminated using an appropriate strategy.

The four elements of the analysis were located in a matrix and evaluated by experts. These results were aggregated using percentages of the assessments. More details of this methodol-

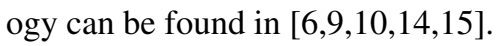

The combination of strengths and opportunities gives rise to potentialities, which indicate the most promising lines of action for the organization. Limitations, determined by a combination of weaknesses and threats, pose a serious warning. Risks (the combination of strengths and threats) and challenges (the combination of weaknesses and opportunities), determined by their corresponding combination of factors, will require careful consideration when setting the course that the organization must take toward the desirable future.

\section{Materials and Methods}

This section describes the algorithm used to evaluate entrepreneurship competence in higher-education students. First, to facilitate the evaluation of the aspects to be measured, two tables relate linguistic terms to plithogenic numbers. These 
plithogenic numbers consist of a vector of three components $(T, I, F)$ such that $T$ represents the degree of truthfulness of what is claimed, $I$ represents the degree of its accuracy, and $F$ represents the degree of its falsehood. $T, I$, and $F$ are the elements of the interval $[0,1]$. Decision-makers make assessments using linguistic terms, which better express what they want to say, and then numerical calculations are carried out with the support of their associated plithogenic numbers. For this reason, this paper uses two tables, Table 1 , which associates linguistic evaluation terms with plithogenic numbers, and Table 2 which associates linguistic terms of weight or importance with plithogenic numbers [18].

Let us denote the set of specialists as $e_{u}\left(u=1,2, \cdots, n_{e}\right)$. Let us suppose that $E_{o_{k} s_{i}}^{u}, E_{t_{l} s_{i}}^{u}, E_{w_{j} o_{k}}^{u}$, and $E_{w_{j} t_{l}}^{u}$ are the evaluations assigned to the intersection of the $k$ th opportunity with the $i$ th strength, the assessment assigned to the intersection of the $l$ th threat with the $i$ th strength, the evaluation assigned to the intersection of the $j$ th weakness with the $k$ th opportunity, and the evaluation assigned to the intersection of the $j$ th weakness with the $l$ th threat, respectively, given by the $u$ th specialist, where $i=1,2, \cdots, n_{s}, j=1,2, \cdots, n_{w}, k=1,2, \cdots, n_{o}$, and $l=1,2, \cdots, n_{t}$.

Additionally, $\omega_{s_{i}}^{u}, \omega_{w_{j}}^{u}, \omega_{o_{k}}^{u}$, and $\omega_{t_{l}}^{u}$ denote the weights or the importance of the $i$ th strength, the $j$ th weakness, the $k$ th opportunity, and the $l$ th threat tofor achieveachieving the goal that the students fulfill the entrepreneurship competence, respectively.

The algorithm designed is the following:

1. To select the group of specialists $e_{u}(u=1,2, \cdots$, $n_{e}$ ) who will assess the entrepreneurship competence or in general any psycho-pedagogical competence or skill. Any method of expert selection can be used for this purpose.

Table 1. Linguistic values associated with plithogenic numbers for the evaluation of students' competence

\begin{tabular}{lc}
\hline Language expression & Plithogenic number $(T, I, F)$ \\
\hline Very poor (VP) & $(0.10,0.75,0.85)$ \\
Poor (P) & $(0.25,0.60,0.80)$ \\
Medium poor (MP) & $(0.40,0.70,0.50)$ \\
Medium (M) & $(0.50,0.40,0.60)$ \\
Medium good (MG) & $(0.65,0.30,0.45)$ \\
Good (G) & $(0.80,0.10,0.30)$ \\
Very good (VG) & $(0.95,0.05,0.05)$ \\
\hline
\end{tabular}

Adapted from Abdel-Basset et al. [18] with the permission of Elsevier.
Table 2. Linguistic values associated with plithogenic numbers for the evaluation of the weight of the criteria

\begin{tabular}{lc}
\hline Language expression & Plithogenic number $(T, I, F)$ \\
\hline Low significance (LS) & $(0.10,0.70,0.80)$ \\
Equal significance (ES) & $(0.30,0.40,0.80)$ \\
Robust significance (RS) & $(0.50,0.40,0.60)$ \\
Very robust significance (VRS) & $(0.70,0.30,0.10)$ \\
Absolute significance (AS) & $(0.90,0.10,0.10)$ \\
\hline
\end{tabular}

Adapted from Abdel-Basset et al. [18] with the permission of Elsevier.

2. To determine the strengths $\left(s_{i}, i=1,2, \cdots, n_{s}\right)$, weaknesses $\left(w_{j}, j=1,2, \cdots, n_{w}\right)$, opportunities $\left(o_{k}, k=1\right.$, $\left.2, \cdots, n_{o}\right)$, and threats $\left(t_{l}, l=1,2, \cdots, n_{t}\right)$ of the organization to analyze entrepreneurship competence or other competencies or skills.

3. Each $e_{u}$ evaluates the combinations Strength-Opportunity, Strength-Threat, Weakness-Opportunity, and WeaknessThreat that organizations may satisfy concerning entrepreneurship competence; thus, $E_{o_{k} s_{i}}^{u}, E_{t_{l} s_{i}}^{u}, E_{w_{j} o_{k}}^{u}$, and $E_{w_{j} t_{l}}^{u}$ are obtained.

In addition, they assess the weight or importance of every aspect. Then, we have $\omega_{s_{i}}^{u}, \omega_{w_{j}}^{u}, \omega_{o_{k}}^{u}$, and $\omega_{t_{l}}^{u}$. In this step, the Delphi method was applied.

For this, experts selected the linguistic terms listed in Tables 1 and 2, and their corresponding plithogenic numbers were used for evaluation. Suppose that $E_{o_{k} s_{i}}^{u}$, $E_{t_{l} s_{i}}^{u}, E_{w_{j} o_{k}}^{u}, E_{w_{j} t_{l}}^{u}, \omega_{s_{i}}^{u}, \omega_{w_{j}}^{u}, \omega_{o_{k}}^{u}$, and $\omega_{t_{l}}^{u}$ are given in the form of plithogenic numbers.

4. To calculate the median of the plithogenic numbers for each combination of Strength-Opportunity, Strength-Threat, Weakness-Opportunity, and Weakness-Threat, for all specialists. The median was calculated using the following formula:

$$
\operatorname{median}_{i=1}^{m}\left\{P N_{i}\right\}=(M T, M I, M F),
$$

where $P N_{i}$ are $m$ plithogenic numbers,

$M T=\operatorname{median}_{i=1}^{m}\left\{T\left(P N_{i}\right)\right\}, \quad M I=\operatorname{median}_{i=1}^{m}\left\{I\left(P N_{i}\right)\right\}$,

and $M F=\operatorname{median}_{i=1}^{m}\left\{F\left(P N_{i}\right)\right\} \cdot T\left(P N_{i}\right)$ are their true components, $I\left(P N_{i}\right)$ are their indeterminate components, and $F\left(P N_{i}\right)$ are their falsehood components. In other words, this equation means that we define the median of 
a set of plithogenic numbers as the plithogenic number of the medians of their components.

We denote by $E_{o_{k} s_{i}}, E_{t_{l} s_{i}}, E_{w_{j} o_{k}}, E_{w_{j} t_{l}}, \omega_{s_{i}}, \omega_{w_{j}}$, $\omega_{o_{k}}$, and $\omega_{t_{l}}$ are the medians of these plithogenic numbers.

That is to say, we obtain:

$E_{o_{k} s_{i}}=\operatorname{median}_{u=1}^{n_{e}}\left\{E_{o_{k} s_{i}}^{u}\right\}, E_{t_{l} s_{i}}=\operatorname{median}_{u=1}^{n_{e}}\left\{E_{t_{l} s_{i}}^{u}\right\}$,

$E_{w_{j} o_{k}}=\operatorname{median} n_{u=1}^{n_{e}}\left\{E_{w_{j} o_{k}}^{u}\right\}$,

and $E_{w_{j} t_{l}}=\operatorname{median}_{u=1}^{n_{e}}\left\{E_{w_{j} t_{l}}^{u}\right\}$.

And also:

$\omega_{s_{i}}=\operatorname{median}_{u=1}^{n_{e}}\left\{\omega_{s_{i}}^{u}\right\}, \omega_{w_{j}}=\operatorname{median}_{u=1}^{n_{e}}\left\{\omega_{w_{j}}^{u}\right\}$,

$\omega_{o_{k}}=\operatorname{median}_{u=1}^{n_{e}}\left\{\omega_{o_{k}}^{u}\right\}$, and $\omega_{t_{l}}=\operatorname{median}_{u=1}^{n_{e}}\left\{\omega_{t_{l}}^{u}\right\}$.

5. To design the SWOT matrix using the aggregated values for all the specialists; i.e., $E_{o_{k} s_{i}}, E_{t_{l} s_{i}}, E_{w_{j} o_{k}}, E_{w_{j} t_{l}}$, $\omega_{s_{i}}, \omega_{w_{j}}, \omega_{o_{k}}$, and $\omega_{t_{l}}$.

6. To calculate the aggregation of the values for every quadrant, Strength-Opportunity (SO), Strength-Threat (ST), Weakness-Opportunity (WO), and Weakness-Threat (WT) by using formulas $(8),(9),(10),(11)$, and $(12)$.

$$
S O=\operatorname{mean}\left\{S O_{L}, S O_{U}\right\}
$$

where $S O$ denotes the aggregated value obtained in the quadrant Strength-Opportunity,

$$
\begin{aligned}
& S O_{L}=\wedge_{p_{k=1}}^{n_{o}} \wedge_{p_{i=1}}^{n_{s}}\left(\left(\omega_{o_{k}} \wedge_{p} \omega_{s_{i}}\right) \wedge_{p} E_{o_{k} s_{i}}\right), \\
& S O_{U}=\vee_{p_{k=1}}^{n_{o}} \vee_{p_{i=1}}^{n_{s}}\left(\left(\omega_{o_{k}} \wedge_{p} \omega_{s_{i}}\right) \wedge_{p} E_{o_{k} s_{i}}\right) \text {, }
\end{aligned}
$$

and $\wedge_{p}$ is the plithogenic neutrosophic intersection based on the minimum $t$-norm and the maximum $t$-conorm (see Equations (6) and (7)), whereas $v_{p}$ is the plithogenic neutrosophic union. Similar to the median, the mean of the two plithogenic numbers is the plitogenic number of the means of every component.

$$
S T=\operatorname{mean}\left\{S T_{L}, S T_{U}\right\},
$$

where $S T$ denotes the aggregated value obtained in the quadrant Strength-Threat,

$$
S T_{L}=\wedge_{p_{i=1}}^{n_{s}} \wedge_{p l=1}^{n_{t}}\left(\left(\omega_{t_{l}} \wedge_{p} \omega_{s_{i}}\right) \wedge_{p} E_{t_{l} s_{i}}\right) \text {, and }
$$

$$
\begin{gathered}
S T_{U}=\vee_{p_{i=1}}^{n_{s}} \vee_{p_{l=1} n_{t}}\left(\left(\omega_{t_{l}} \wedge_{p} \omega_{s_{i}}\right) \wedge_{p} E_{t_{l} s_{i}}\right) . \\
W O=\operatorname{mean}\left\{W O_{L}, W O_{U}\right\},
\end{gathered}
$$

where $W O$ denotes the aggregated value obtained in the quadrant Weakness-Opportunity,

$$
\begin{aligned}
& W O_{L}=\wedge_{p_{j=1}}^{n_{w}} \wedge_{p_{k=1}}^{n_{o}}\left(\left(\omega_{w_{j}} \wedge_{p} \omega_{o_{k}}\right) \wedge_{p} E_{w_{j} o_{k}}\right) \text {, and } \\
& W O_{U}=\vee_{p_{j=1}}^{n_{w}} \vee_{p_{k=1}}^{n_{o}}\left(\left(\omega_{w_{j}} \wedge_{p} \omega_{o_{k}}\right) \wedge_{p} E_{w_{j} o_{k}}\right) \text {. } \\
& W T=\operatorname{mean}\left\{W T_{L}, W T_{U}\right\},
\end{aligned}
$$

where $W T$ denotes the aggregated value obtained in quadrant Weakness-Threat,

$$
\begin{aligned}
& W T_{L}=\wedge_{p_{j=1}}^{n_{w}} \wedge_{p_{l=1}}^{n_{t}}\left(\left(\omega_{w_{j}} \wedge_{p} \omega_{t_{l}}\right) \wedge_{p} E_{w_{j} t_{l}}\right), \text { and } \\
& W T_{U}=\vee_{p_{j=1}}^{n_{w}} \vee_{p_{l=1}}^{n_{t}}\left(\left(\omega_{w_{j}} \wedge_{p} \omega_{t_{l}}\right) \wedge_{p} E_{w_{j} t_{l}}\right) .
\end{aligned}
$$

7. To compare $S O, S T, W O$, and $W T$ with the plithogenic numbers in Table 1 by using Equation (13):

$$
d\left(Q, T V_{v}\right)=\sqrt{\left(Q-T V_{v}\right) \cdot\left(Q-T V_{v}\right)^{\prime}},
$$

where $Q$ denotes the plithogenic number in the quadrant, $T V_{v}$ is the plithogenic number of the $v$ th member of Table 1, $T(\cdot), I(\cdot)$, and $F(\cdot)$ are their truth, indeterminate, and falseness values, respectively, and ' is the matrix transpose operator.

Thus, by fixing $Q$, we select $\widehat{v}=\min _{v=1}^{7}\left\{d\left(Q, T V_{v}\right)\right\}$, and we evaluate $Q$ with the $\widehat{v} t h$ linguistic term in Table 1

Hence, the linguistic evaluation of $S O$ is utilized to assess the potentialities, $S T$ the risks, $W O$ the challenges, and $W T$ the limitations.

8. To compare the relationships among the quadrants, we use the following formula to fuzzify a neutrosophic number [18]:

$$
\mathcal{S}([T, I, F])=\frac{2+T-I-F}{3} .
$$

Then, calculate $v_{D}=\mathcal{S}(S O), v_{1}=\mathcal{S}(S T), v_{2}=$ $\mathcal{S}(W O)$, and $v_{3}=\mathcal{S}(W T)$. As can be seen, we recommend selecting the $S O$ as the dominant attribute value.

9. To define the attribute value contradiction degree function 
$c_{F}$, we recommend the following a priori values:

$$
\begin{aligned}
& c(S O, S T)=0.5, c(S O, W O)=0.5, \text { and } \\
& c(S O, W T)=1 .
\end{aligned}
$$

Any other attribute value contradiction degree function can be defined up to the specialists' interests.

10. To apply Formula (3) for every pair, viz. $v_{D}$ and $v_{1}, v_{D}$ and $v_{2}, v_{D}$ and $v_{3}$.

These results are interpreted as follows:

I) If the value is $>0.5$ we say that the truth-value representing $\mathrm{SO}$ and $v_{p}(p=1,2,3)$ is "sufficiently high", and:

(i) The institution has to face "sufficiently high" potentialities and risks if $p=1$.

(ii) The institution has to face "sufficiently high" potentialities and challenges if $p=2$.

(iii) The institution has to face "sufficiently high" potentialities and limitations if $p=3$.

II) If the value is $=0.5$ we say that the truth-value representing $\mathrm{SO}$ and $v_{p}(p=1,2,3)$ is "as high as low", and:

(i) The institution has to face "as high as low" potentialities and risks if $p=1$.

(ii) The institution has to face "as high as low" potentialities and challenges if $p=2$.

(iii) The institution has to face "as high as low" potentialities and limitations if $p=3$.

III) If the value is $<0.5$ we say that the truth-value representing $\mathrm{SO}$ and $v_{p}(p=1,2,3)$ is "sufficiently low", and:

(i) The institution has to face "sufficiently low" potentialities and risks if $p=1$.

(ii) The institution has to face "sufficiently low" potentialities and challenges if $p=2$.

(iii) The institution has to face "sufficiently low" potentialities and limitations if $p=3$.

Figure 1 summarizes the steps of this algorithm.

From the points listed below, it can be inferred that the use of a model based on plithogenic numbers is acceptable for measuring entrepreneurship competence:

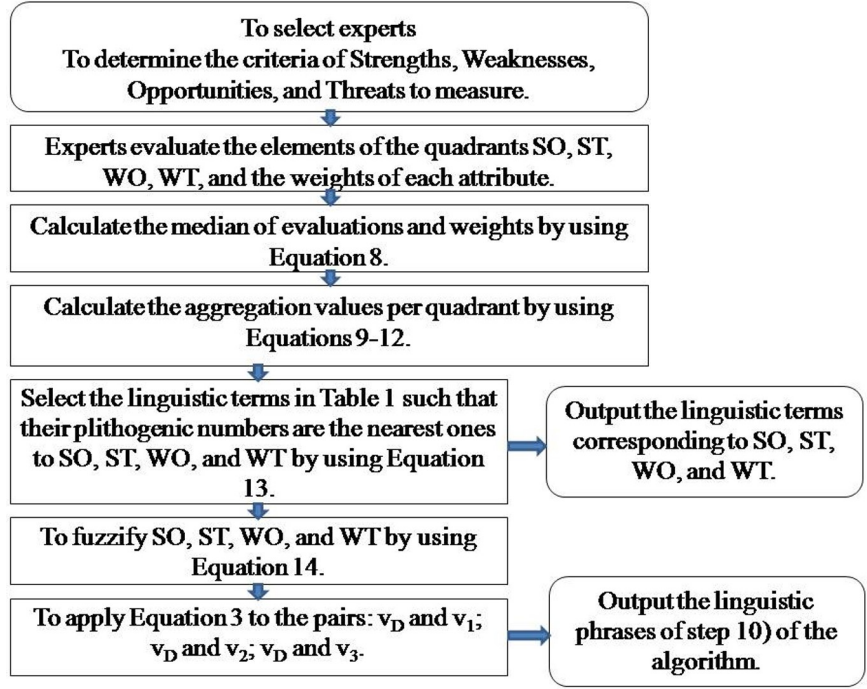

Figure 1. Scheme of the proposed algorithm.

1. The measurement scale using linguistic terms associated with plithogenic numbers has been effectively applied in other similar problems to measure situations where humans express opinions, see [18].

2. Plithogenic numbers explicitly take into account the indeterminacy symbolized the component $I$. This component does not exist in fuzzy theory and is limited to the intuitionistic fuzzy set theory. This is inherited from the neutrosophy set theory which allows us to express some kinds of knowledge and information that exists in the social sciences; for example, paradoxes can be represented by the triple $(1, I, 1)$, where the proposition is both true and false; the property $t+i+f<1$ indicates that there is incomplete information, whereas $t+i+f>1$ indicates contradictory information. This flexibility to express these states of knowledge is not possible by using fuzzy logic or intuitionistic fuzzy logic [19].

3. Operations with plithogenic numbers correspond to the so-called Zimmermann's Compensatory AND [20]. This author experimentally demonstrated that formula (2) corresponds more adequately with the way humans aggregate AND, when compared with the $t$-norm.

4. The specialists surveyed at the University of Guayaquil were satisfied with the model and its results. It is necessary to apply it successfully to a greater number of problems to ensure the validity of this model. 


\section{Case Study of the Students at the University of Guayaquil}

In this section, we discuss the case study at the University of Guayaquil. To provide greater objectivity to this feasibility analysis in the application of a strategy for the development of entrepreneurship competence in the students of the University of Guayaquil, the authors assume that actions cannot defect to their factual contextualization.

Thus, we selected 11 specialists from the University of Guayaquil, who knows well the characteristics of the students of this university, to assess these students on entrepreneurship competence, according to the following aspects:

The aspects of the external analysis in terms of opportunities are as follows:

O1. The social demand is characterized by requiring from university students that they were entrepreneurs and have a good development in lateral thinking.

O2. The Eeconomic Mmodel inferred by the Ecuadorian legal system advocates a change of the productive matrix.

The analyzed threat aspects are the following:

T1. There is a lack of formative intentionality of the curriculum system that difficult the development of entrepreneurship competence in the students of the University of Guayaquil.

T2. There is no implementation of competency training in the Ecuadorian educational system and especially in university education.

The aspects of the internal analysis regarding weaknesses are as follows:

W1. There is insufficient planning, organization, execution, and control of research projects.

W2. There is poor interpretation and understanding of social reality and the environment where students live and grow up.

W3. There are very few opportunities to establish judgments and reasoning in the comprehensive general culture from the knowledge, skills, and values of the students.

W4. There is underdevelopment of creativity from the teachinglearning process, it appeals to reproductive strategies of the acquired knowledge, which diminishes their initiatives.
W5. There is poor progress of critical and lateral thinking, low holistic understanding of the social environment and its demands.

W6. There is a meager development of resilience in confronting academic problems by students, revealed in interviews, curriculum analysis, and spaces for reflection and debate.

W7. There is underdevelopment of the value responsibility in the student, for the teaching and extra-docent tasks.

W8. The preponderance of social interest, equity, and respect for differences by students in working in groups within the class is not significant.

W9. There is insufficient perception of the value of nature, sustainable development, and environmental care by students.

The aspects of the internal analysis in terms of strengths are the following:

S1. There is an adequate learning development of technical knowledge, skills, and values in students.

S2. There is a good use of tools and technologies for information management.

S3. There is a high willingness to leadership and decisionmaking, from the training processes.

S4. There is an excellent performance in the team work, which is evidenced in the appropriation and collaboration to obtain knowledge.

S5. Students' optimism about their professional future and prospects for employability is valued as good.

Among the consulted specialists, there are managers such as deans of faculties, specialists in human resources, and prestigious professors within the institution. Each of them used Tables 1 and 2 to provide their assessments based on linguistic terms. The equivalent plithogenic numbers were determined for each aspect to be evaluated.

The specialists assigned a weight to each of the measured aspects of the matrix, according to the terms in Table 2 , and then they were aggregated with the median, using their plithogenic numbers, which is reflected in Table 3

Additionally, for each aspect to be measured, the median of the plithogenic numbers corresponding to the evaluations of all experts was calculated to obtain a unique value for each 
Table 3. Neutrosophic numbers obtained from calculating the median of the weights evaluated by the specialists

\begin{tabular}{cc}
\hline Aspect to measure & $\begin{array}{c}\text { Aggregated plithogenic } \\
\text { numbers of specialists }\end{array}$ \\
\hline O1 & $(0.90,0.10,0.10)$ \\
O2 & $(0.90,0.10,0.10)$ \\
T1 & $(0.70,0.30,0.10)$ \\
T2 & $(0.70,0.30,0.10)$ \\
\hline S1 & $(0.90,0.10,0.10)$ \\
\hline S2 & $(0.70,0.30,0.10)$ \\
\hline S3 & $(0.70,0.30,0.10)$ \\
\hline S4 & $(0.70,0.30,0.10)$ \\
\hline S5 & $(0.50,0.40,0.60)$ \\
\hline W1 & $(0.70,0.30,0.10)$ \\
\hline W2 & $(0.70,0.30,0.10)$ \\
\hline W3 & $(0.90,0.10,0.10)$ \\
\hline W4 & $(0.90,0.10,0.10)$ \\
\hline W5 & $(0.70,0.30,0.10)$ \\
\hline W6 & $(0.50,0.40,0.60)$ \\
\hline W7 & $(0.70,0.30,0.10)$ \\
\hline W8 & $(0.70,0.30,0.10)$ \\
\hline W9 & $(0.70,0.30,0.10)$ \\
\hline
\end{tabular}

aspect. In Table 4 , these results are represented in the form of the aggregated plithogenic numbers.

To calculate the results of the aggregation by the four quadrants, namely SO, ST, WO, and WT, formulas 99, (10), 111, and (12) are used, respectively. In this study, we do not differentiate any dominant aspect of these formulas.

Applying the results obtained from Tables 3 and 4 , and Equations (6) and (7), we have

$$
\begin{aligned}
S O & =\text { mean }\{(0.50,0.15527,0.60),(0.90,0.15527,0.10)\} \\
& =(0.70,0.15527,0.35), \\
S T & =\text { mean }\{(0.25,0.32305,0.80),(0.65,0.32305,0.45)\} \\
& =(0.45,0.32305,0.625), \\
W O & =\text { mean }\{(0.25,0.28748,0.80),(0.80,0.28748,0.30)\} \\
& =(0.525,0.28748,0.55), \text { and } \\
W T & =\text { mean }\{(0.50,0.28748,0.60),(0.8,0.28748,0.3)\} \\
& =(0.65,0.28748,0.45) .
\end{aligned}
$$

After consulting Table 1, we find that by similarity using
Equation (13) and step 7) of the algorithm, we can qualify both, ST (with distance 0.095112) and WO (with distance 0.12564) as "Medium", whereas SO is "Good" (with distance 0.12472) and WO is "Medium good" (with distance 0.012520).

To further deepen, the four plithogenic numbers are converted into fuzzy values using Equation (14).

Thus, $v_{D}=\mathcal{S}(S O)=0.73158, v_{1}=\mathcal{S}(S T)=0.50065$, $v_{2}=\mathcal{S}(W O)=0.56251$, and $v_{3}=\mathcal{S}(W T)=0.63751$.

Then, we apply formula (3) with the $t$-norm and $t$-conorm used, and we obtain the following results:

$$
\begin{aligned}
& {[1-c(S O, S T)] \cdot\left(S O \wedge_{F} S T\right)+c(S O, S T) \cdot\left(S O \vee_{F} S T\right)} \\
& =\quad(1-0.5) \min (0.73158,0.50065) \\
& \quad+0.5 \max (0.73158,0.50065)=0.61611 . \\
& {[1-c(S O, W O)] \cdot\left(S O \wedge_{F} W O\right)} \\
& \quad+c(S O, W O) \cdot\left(S O \vee_{F} W O\right)=0.64704, \text { and } \\
& {[1-c(S O, W T)] \cdot\left(S O \wedge_{F} W T\right)} \\
& \quad+c(S O, W T) \cdot\left(S O \vee_{F} W T\right)=0.73158 .
\end{aligned}
$$

Because these values are greater than 0.5 , we can say that there are "sufficiently high" potentialities and risks, "sufficiently high" potentialities and challenges, and "sufficiently high" potentialities and limitations to achieve the goal of entrepreneurship competence.

\section{Discussion}

Entrepreneurship competence is understood as a system of elements that interact with each other for the formation development of a student who should be able to self-regulate and actively influence his/her formation, and in this way, they become professionals that today's society needs.

One of the purposes of this research is to provide educators with a rigorous logical-mathematical tool that allows them to measure the situation of this competence or other abilities of university students to improve their competence. Such a method should have the following characteristics:

1. It is simple, preferably on a measuring scale that is easy to use by specialists.

2. It captures the complexity of the phenomenon that is measured.

3. It includes the uncertainty and imprecision of this type of concept. 
Table 4. SWOT matrix of the aspects measured in the form of plithogenic numbers

\begin{tabular}{|c|c|c|c|c|c|}
\hline & \multirow{2}{*}{ No. } & \multicolumn{2}{|c|}{ Opportunities } & \multicolumn{2}{|c|}{ Threats } \\
\hline & & $\mathrm{O} 1$ & $\mathrm{O} 2$ & $\mathrm{~T} 1$ & $\mathrm{~T} 2$ \\
\hline \multirow[t]{5}{*}{ Strengths } & $\mathrm{S} 1$ & $(0.95,0.05,0.05)$ & $(0.95,0.05,0.05)$ & $(0.65,0.30,0.45)$ & $(0.65,0.30,0.45)$ \\
\hline & $\mathrm{S} 2$ & $(0.95,0.05,0.05)$ & $(0.95,0.05,0.05)$ & $(0.50,0.40,0.60)$ & $(0.50,0.40,0.60)$ \\
\hline & S3 & $(0.80,0.10,0.30)$ & $(0.95,0.05,0.05)$ & $(0.40,0.70,0.50)$ & $(0.50,0.40,0.60)$ \\
\hline & $\mathrm{S} 4$ & $(0.80,0.10,0.30)$ & $(0.80,0.10,0.30)$ & $(0.65,0.30,0.45)$ & $(0.65,0.30,0.45)$ \\
\hline & S5 & $(0.95,0.05,0.05)$ & $(0.80,0.10,0.30)$ & $(0.25,0.60,0.80)$ & $(0.50,0.40,0.60)$ \\
\hline \multirow[t]{9}{*}{ Weaknesses } & W1 & $(0.25,0.60,0.80)$ & $(0.50,0.40,0.60)$ & $(0.80,0.10,0.30)$ & $(0.65,0.30,0.45)$ \\
\hline & W2 & $(0.65,0.30,0.45)$ & $(0.65,0.30,0.45)$ & $(0.80,0.10,0.30)$ & $(0.80,0.10,0.30)$ \\
\hline & W3 & $(0.80,0.10,0.30)$ & $(0.80,0.10,0.30)$ & $(0.65,0.30,0.45)$ & $(0.65,0.30,0.45)$ \\
\hline & W4 & $(0.80,0.10,0.30)$ & $(0.65,0.30,0.45)$ & $(0.80,0.10,0.30)$ & $(0.65,0.30,0.45)$ \\
\hline & W5 & $(0.65,0.30,0.45)$ & $(0.80,0.10,0.30)$ & $(0.65,0.30,0.45)$ & $(0.65,0.30,0.45)$ \\
\hline & W6 & $(0.50,0.40,0.60)$ & $(0.50,0.40,0.60)$ & $(0.50,0.40,0.60)$ & $(0.65,0.30,0.45)$ \\
\hline & W7 & $(0.50,0.40,0.60)$ & $(0.50,0.40,0.60)$ & $(0.80,0.10,0.30)$ & $(0.80,0.10,0.30)$ \\
\hline & W8 & $(0.65,0.30,0.45)$ & $(0.65,0.30,0.45)$ & $(0.65,0.30,0.45)$ & $(0.65,0.30,0.45)$ \\
\hline & W9 & $(0.65,0.30,0.45)$ & $(0.65,0.30,0.45)$ & $(0.65,0.30,0.45)$ & $(0.65,0.30,0.45)$ \\
\hline
\end{tabular}

The designed method satisfied all of these characteristics. On the one hand, experts must only evaluate based on a linguistic scale that is very similar to the method of evaluation by any human being. This avoids biases when performing the assessment and ensures greater coincidence between what the expert says and what he/she wants to express. The final result of the method is also given in the form of linguistic terms, allowing them to be easily understood by managers, educators, and students.

On the other hand, the use of plithogenic numbers makes it possible to model a competence that depends on many complex aspects, such as the educational system, the state's attention to students, the social recognition of professionals, the economic system, the access to information, and the excellence of universities where highly professional specialists are hired. Plithogeny is a novel theory that allows modeling complex systems obtained from the interaction, sometimes nonlinear, among simpler components, not necessarily free of contradictions, to obtain a new element. Plithogenic numbers based on neutrosophy contain within their definition not only the uncertainty of other logics but also the indeterminacy that is part of neutrosophy.

In this method, plithogenic numbers are hybridized with SWOT analysis, which permits the precise determination of tactics and strategies to follow to improve the situation of this or other pedagogical competence.

A complex system is composed of interrelated parts that exhibit non-evident properties and behaviors from the sum of the individual parts. The characteristics of complex systems (such as interdependence, diversity, and adaptability of agents) challenge the basic assumptions of traditional theories.

Complex phenomena are characterized by interactions in the form of nonlinear and sometimes chaotic dynamics of simpler elements. The plithogenic theory aims to model this type of phenomenon, which is deduced from its definition. Rarely, complex phenomena can be logically evaluated with a logic that expresses a $100 \%$ of truthfulness, which is why uncertainty models such as probabilistic models or those based on fuzzy logic or its generalizations (intuitionistic fuzzy logic or neutrosophic logic), are needed. Furthermore, plithogenic logic generalizes the aforementioned logic and allows us to model the interactions of more than one idea, theory, concept, etc.

Apart from this, plithogenic theory allows us to model confused, unknown, incomplete, paradoxical, incoherent, and contradictory ideas or situations, which is what is called imprecision. This imprecision can be represented numerically and symbolically by symbol $I$, which is not possible with other logic. The most approached theory to plithogenic logic is neutrosophic logic, which only allows us to model the dynamic interaction between the truthfulness, neutrality, and falsity of a single concept, whereas plithogeny generalizes it to the interaction between different concepts, ideas, theories, and so on. 
In addition, the SWOT method with its four components also permits a simple approach to the complexity of a social situation; for example, in pedagogical sciences, it has been used in problems as dissimilar as can be consulted in [21-24].

Comparing this method with the classical crisp SWOT analysis, we find that the latter method is the simpler one; however, it does not take into account the uncertainty or imprecision of the assessment. Moreover, because we are modeling with logical propositions, the way to evaluate the components of each quadrant can be made by assessing some specifically logical propositions; for example, instead of using elements in Equation 9, we can use other more complex equations, but which are more interesting, as follows:

$$
\begin{aligned}
& S O_{L}=\wedge_{p}^{n_{o}} \wedge_{k=1}^{n_{s}}\left(\left(\omega_{i=1} \wedge_{p} E_{o_{k}}\right) \wedge_{p}\left(\omega_{s_{i}} \wedge_{p} E_{s_{i}}\right)\right), \\
& S O_{U}=\vee_{p_{k=1}^{n_{o}}}^{\vee_{p} n_{i=1}}\left(\left(\omega_{o_{k}} \wedge_{p} E_{O_{k}}\right) \wedge_{p}\left(\omega_{s_{i}} \wedge_{p} E_{s_{i}}\right)\right),
\end{aligned}
$$

where $E_{o_{k} s_{i}}$ is divided into $E_{o_{k}}$ to evaluate the $k$ th opportunity $o_{k}$, and $E_{s_{i}}$ denotes the evaluation of the $i$ th strength. With this formula, experts do not have to provide only one value for measuring two different aspects.

Future analysis of the limitations of the method is to analyze Equations (9), (10), (11), and (12) to find other ways to measure each quadrant that isare more effective than those used. It is worth noting that the applied formulas are based on the deneutrosophication [25], of an interval where the extremes are the sums of the sum, as shown in Equation (15).

$$
\lambda([a, b])=\frac{a+b}{2} .
$$

In future work, the authors propose to apply the method in other case studies of entrepreneurship competence and other skills to further validate the method. We propose to enrich the method by including the Delphi method (see, for example [26]) hybridized with the SWOT analysis for expert evaluation.

\section{Conclusion}

In this study, we designed a new method to measure entrepreneurship competence among higher-education students. The method is simple to apply, and its inputs and outputs are given in the form of natural language; thus, the results and data are comprehensible to the experts, managers, professors, and students. The method was applied successfully to a case study of students at the University of Guayaquil in Ecuador. The method combines the advantages of using both the SWOT method and plythogenic theory, and thus, it includes the uncertainty and imprecision typical of every complex phenomenon of social sciences. To the best of our knowledge, this is the first time that plithogeny is applied in a pedagogical study of any competence or skill.

\section{Conflict of Interest}

No potential conflict of interest relevant to this article was reported.

\section{References}

[1] N. Batista Hernandez, N. Valcárcel Izquierdo, G. Real Zumba, and A. D. Alban Navarro, "Development of the entrepreneurship competition, a necessity in the integral formation of the student," Dilemas Contemporáneos: Educación, Política y Valores, vol. 5, no. 1, article no. 36, 2017. https: //www.dilemascontemporaneoseducacionpoliticayvalores. com/index.php/dilemas/article/view/137

[2] N. B. Hernandez, W. O. Aguilar, and J. E. Ricardo, "Local development and training of the pedagogical competition of entrepreneurship: a need in the social context of Cuba," Didasc@lia: Didácticay Educación, vol. 8, no. 5,pp. 213-226, 2017.

[3] N. B. Hernandez, R. V. G. Intriago, J. C. G. Espinoza, and P. J. D. Vasconez, "Competition of entrepreneurship as a support for integral training and social inclusion of the student," Revista órbita pedagógica, vol. 4, no. 3, pp. $115-125,2018$

[4] N. B. Hernandez and N. V. Izquierdo, "Theoretical validation of the strategy for the development of competence engage as a contribution to the integral training of the student of pre-university education," Magazine de las Ciencias, vol. 3, no. 3, pp. 103-116, 2018.

[5] D. A. Kirby, "Entrepreneurship education: can business schools meet the challenge?," Education+ Training, vol. 46, no. 8-9, pp. 510-519, 2004. https://doi.org/10.1108/ 00400910410569632

[6] L. K. B. Villanueva, D. A. V. Intriago, L. K. A. Gomez, and A. M. I. Moran, "Business plan for entrepreneurs, actors and organizations of social and solidarity economy based on 
neutrosophic AHP-SWOT,' Neutrosophic Sets and Systems, vol. 37, no. 1, pp. 224-234, 2020.

[7] J. E. Ricardo, M. A. V. Coloma, A. T. C. Maldonado, and L. A. C. Hurtado, "Reflections on the relevance and impact of higher education in ecuador from its current perspective," Revista de Entrenamiento, vol. 3, no. 3, pp. 81-92, 2018.

[8] B. Fernandez Oliva, I. Morales Suarez, and J. Portal Pineda, "System of influences for the integral formation of the graduated of the centers of Superior Medical Education [Sistema de influencias para la formación integral de los egresados de los centros de Educación Médica Superior]," Educación Médica Superior, vol. 18, no. 2, 2014.

[9] T. Hill and R. Westbrook, "SWOT analysis: it's time for a product recall," Long Range Planning, vol. 30, no. 1, pp. 4652, 1997. https://doi.org/10.1016/S0024-6301(96)00095-7

[10] G. Houben, K. Lenie, and K. Vanhoof, "A knowledgebased SWOT-analysis system as an instrument for strategic planning in small and medium sized enterprises," Decision Support Systems, vol. 26, no. 2, pp. 125-135, 1999. https: //doi.org/10.1016/S0167-9236(99)00024-X

[11] F. Smarandache, Plithogeny, Plithogenic Set, Logic, Probability, and Statistics. Brussels, Belgium: Pons Publishing, 2017.

[12] F. Smarandache, "Plithogenic set, an extension of crisp, fuzzy, intuitionistic fuzzy, and neutrosophic sets-revisited," Neutrosophic Sets and Systems, vol. 21, pp. 153-166, 2018.

[13] M. Abdel-Basset, R. Mohamed, A. E. N. H. Zaied, and F. Smarandache, "A hybrid plithogenic decision-making approach with quality function deployment for selecting supply chain sustainability metrics," Symmetry, vol. 11, no. 7, article no. 903, 2019. https://doi.org/10.3390/sym11070903

[14] M. Abdel-Basset, M. Mohamed, and F. Smarandache, "An extension of neutrosophic AHP-SWOT analysis for strategic planning and decision-making," Symmetry, vol. 10, no. 4, article no. 116, 2018. https://doi.org/10.3390/ sym 10040116

[15] M. A. Basset, M. Mohamed, A. K. Sangaiah, and V. Jain, "An integrated neutrosophic AHP and SWOT method for strategic planning methodology selection," Benchmarking: An International Journal, vol. 25, no. 7, pp. 2546-2564, 2018. https://doi.org/10.1108/BIJ-08-2017-0232
[16] M. Grida, R. Mohamed, and A. N. H. Zaied, "A novel plithogenic MCDM framework for evaluating the performance of IoT based supply chain," Neutrosophic Sets and Systems, vol. 33, no. 1, pp. 323-341, 2020.

[17] E. P. Klement, R. Mesiar, and E. Pap, Triangular Norms. Dordrecht, The Netherlands: Springer, 2000.

[18] M. Abdel-Basset, M. El-Hoseny, A. Gamal, and F. Smarandache, "A novel model for evaluation Hospital medical care systems based on plithogenic sets," Artificial Intelligence in Medicine, vol. 100, article no. 101710, 2019. https://doi.org/10.1016/j.artmed.2019.101710

[19] C. Ashbacher, Introduction to Neutrosophic Logic. Rehoboth, NM: American Research Press, 2002.

[20] H. J. Zimmermann and P. Zysno, "Latent connectives in human decision making," Fuzzy Sets and Systems, vol. 4, no. 1, pp. 37-51, 1980. https://doi.org/10.1016/01650114(80)90062-7

[21] H. Dixit and S. S. Marahatta, "Medical education and training in Nepal: SWOT analysis," Kathmandu University Medical Journal, vol. 6, no. 3, pp. 412-420, 2008. https: //doi.org/10.3126/kumj.v6i3.1725

[22] S. Thomas, Q. T. Chie, M. Abraham, S. Jalarajan Raj, and S. Beh, "A qualitative review of literature on peer review of teaching in higher education: an application of the SWOT framework," Review of Educational Research, vol. 84, no. 1, pp. 112-159, 2014. https://doi.org/10.3102\% 2F0034654313499617

[23] M. K. Shahijan, S. Rezaei, and C. N. Preece, "Developing a framework of internationalisation for higher education institutions in Malaysia: a SWOT analysis," International Journal of Management in Education, vol. 10, no. 2, pp. 145-173, 2016. https://doi.org/10.1504/IJMIE.2016.075556

[24] G. J. Longhurst, D. M. Stone, K. Dulohery, D. Scully, T. Campbell, and C. F. Smith, "Strength, weakness, opportunity, threat (SWOT) analysis of the adaptations to anatomical education in the United Kingdom and Republic of Ireland in response to the Covid-19 pandemic," Anatomical Sciences Education, vol. 13, no. 3, pp. 301-311, 2020. https://doi.org/10.1002/ase.1967

[25] F. Smarandache, Introduction to Neutrosophic Statistics. Craiova, Romaina: Sitech \& Education Publishing, 2014. 
[26] F. Smarandache, J. E. Ricardo, E. G. Caballero, and M. Y. L. Vazquez, and N. B. Hernandez, "Delphi method for evaluating scientific research proposals in a neutrosophic environment," Neutrosophic Sets and Systems, vol. 34, no. 1, pp. 204-213, 2020.

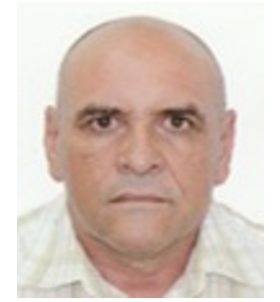

Noel Batista Hernández received a Ph.D. in Pedagogical Sciences and Master's degree in Public Administration, and is a Professor of the University of Guayaquil Ecuador. His research interests focus on topics related to pedagogical training in competitions and analysis of the effectiveness of public management, and an associate editor of the Journal Neutrosophic Computing and Machine Learning.

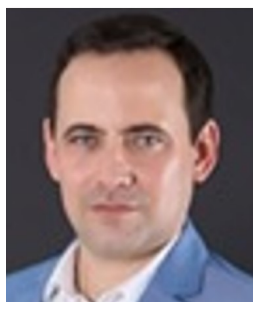

Maikel Y. Leyva Vázquez received his M.Sc. in Bioinformatics in 2007 and his Ph.D. in Technical Sciences in 2013. He has worked as a lecturer in different universities in Cuba and Ecuador. Editor of the Journal Neutrosophic Computing and Machine Learning. His research interests include artificial intelligence, neutrosophy, and machine learning.

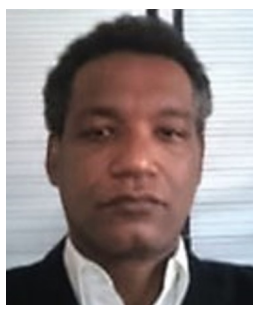

Erick González Caballero received his Ph.D. degree (2013) in technical sciences from the Technological University of Havana, and the B.S. degree (1998) in Mathematics from the University of Havana in Cuba. After graduating, he works as a scientist or teacher at various universities and research centers in Cuba. In 2011, he continued his teaching and scientific activities at the Technological University of Havana. He was a Postdoctoral Visitor (2016) at the University of Oviedo, Spain. His research interests include fuzzy logic, neutrosophy, and cooperative game theory.

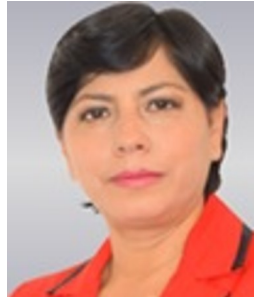

Lilia Esther Valencia Cruzaty received a Ph.D. in Administrative Sciences and Master's degree in Business Administration, more than 30 years dedicated to teaching. Recent work experience includes the rector of the State University Peninsula of Santa Elena, dean of postgraduate University of the Pacific, and director of several international research. Her lines of research are on topics related to education, administration, and entrepreneurship.

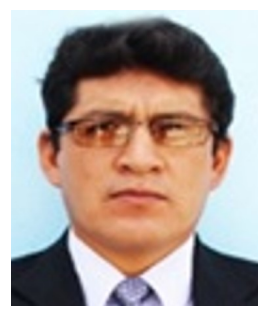

Wilmer Ortega Chávez is a Professor at the National Intercultural University of Amazon Post-Doctor in Philosophy, a Doctor in Environment and Sustainable Development, a Researcher of the International and National Lecturer, an Advisor and Consultant in Quality Management, Regional President of Ucayali of the International Chamber of Speakers, a Member of the Hispanic Society of Researchers of Peru-Mexico; currently working at BIOESTADISTICO and CIGPC consulting manager.

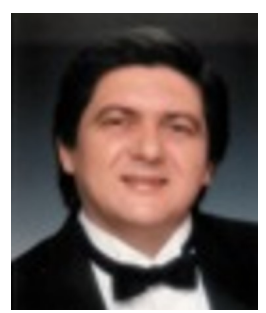

Florentin Smarandache received the M. Sc. degree in mathematics and computer science from the University of Craiova, Romania, and the Ph.D. degree in mathematics from the State University of Kishinev. He held a postdoctoral position in Applied Mathematics at Okayama University of Sciences, Japan. He is currently a professor of mathematics at the University of New Mexico, Gallup, USA. He has been the founder of neutrosophy, neutrosophic over-under-off-sets, plithogenic sets, and many others. 